\title{
Vasorreactividad a adenosina en los pacientes con hipertensión pulmonar arterial: Prevalencia y respuesta clínica, funcional y hemodinámica al tratamiento con diltiazem
}

\author{
MÓNICA ZAGOLÍN B.*, KAREN CZISCHKE L.**, POLENTZI URIARTE G. DE C.***, \\ NICOLÁS MEDEL F.****, CLAUDIO PARRA R.*****, \\ DIEGO VARGAS B.****** y GABRIEL CAVADA CH.*******
}

Acute vasodilatador test using adenosine in patients with pulmonary arterial hypertension: Prevalence and clinical, functional and hemodynamic response after diltiazem treatment

Pulmonary arterial hypertension (PAH) is a severe and progressive disease. Invasive hemodynamic study (HS) is required to confirm the diagnosis of PAH and to perform the vasodilator test (VT) with adenosine. Vasodilator acute responders (VAR) may have a sustained benefit with diltiazem. There is not national information regarding these issues. All patients with probable PAH were evaluated with HS and VT. VAR were treated with diltiazem and followed up with functional class score (FC) and 6 minute walk test. After 6 months, a second HS was performed. Results: Between 2003 and 2008, 6/54 (11\%) were VAR. All were women, $45 \pm 14$ years old, 4 with idiopathic PAH, 4 in FCIII and 2 in FCII. After two years of treatment, all patients clinically improved. Walked distance significantly increased by 83 and $87 \mathrm{~m}$ at month 12 and 24 respectively. Hemodynamic parameters also improved. Therapy with diltiazem is effective in VAR patients supporting the convenience to perform the VT.

Key words: Pulmonary arterial hypertension, vasodilator challenge, calcium channel blockers, diltiazem.

\section{Resumen}

La Hipertensión Pulmonar Arterial (HAP) es una entidad grave y progresiva. El estudio hemodinámica (EH) permite certificar el diagnóstico y evaluar la vasorreactividad mediante adenosina. Los pacientes vaso-reactivos podrían responder a terapia con diltiazem. No existe información nacional al respecto. En nuestro programa todo paciente con sospecha de HAP es sometido a EH diagnóstico y de vasorreactividad. Los positivos son tratados con diltiazem y seguidos semestralmente según capacidad funcional $(C F)$ y con test de caminata. Al $6^{o}$ mes se efectúa un $2^{\circ}$ EH. Entre 2003-2008, 6/54 (11\%) de los pacientes con HAP fueron vasorreactivos. Todas mujeres, $45 \pm 14$ años, 4 con HPA idiopática, 4 en CFIII y 2 en CFII. A los 2 años, todos mejoraron clínicamente. La distancia recorrida aumentó significativamente en los meses 12 y 24 en 83 y 87 m respectivamente. Todas las variables hemodinámicas mejoraron. La terapia con diltiazem es efectiva en los pacientes vaso-reactivos lo que justifica usar el test de vasorreactividad.

Palabras clave: Hipertensión pulmonar, respuesta aguda a vasodilatadores, bloqueadores canales de calcio, diltiazem.

\footnotetext{
* Instituto Nacional del Tórax y Departamento de Medicina, Facultad de Medicina, Universidad de Chile.

** $\quad$ Médico Becado en el Instituto Nacional del Tórax.

*** Departamento de Cardiología del Instituto Nacional del Tórax

**** Interno de Medicina de $7^{\circ}$ año, Facultad de Medicina de la Universidad de Chile.

****** Sección Cardiología, Hospital del Salvador.

****** Kinesiólogo, Instituto Nacional del Tórax.

******* División de Bioestadística, Escuela de Salud Pública de la Facultad de Medicina de la Universidad de Chile.
} 


\section{Introducción}

La hipertensión arterial pulmonar (HAP) es una entidad grave, progresiva y devastadora. Afecta principalmente a mujeres jóvenes, limitando significativamente su capacidad funcional en las edades más productivas. La sobrevida estimada promedio es de 2,8 años sin terapia ${ }^{1}$ aunque depende de los factores pronósticos presentes al momento del diagnóstico. Dentro de ellos, tienen una mejor correlación con la sobrevida la capacidad funcional inicial, la etiología de la HAP, la distancia recorrida en el test de caminata y las variables hemodinámicas documentadas durante el cateterismo derecho así como la respuesta aguda a los vasodilatadores ${ }^{2-4}$.

El diagnóstico de certeza de la HAP es hemodinámico, mediante cateterismo derecho. Se define HAP ante la presencia de una presión de arteria pulmonar media (PAPM) superior a 25 $\mathrm{mmHg}$ con una presión de enclavamiento pulmonar inferior a $15 \mathrm{mmHg}^{5-7}$. Se recomienda con alto nivel de evidencia ${ }^{7}$ efectuar durante el procedimiento hemodinámico diagnóstico una prueba de respuesta aguda a vasodilatadores (prueba de vasorreactividad) para evaluar la factibilidad de respuesta a bloqueadores de canales de calcio (BCC). Las drogas aprobadas para dicho propósito son: adenosina, óxido nítrico o epoprostenol endovenoso ${ }^{7-9}$. Los criterios de respuesta aguda a estas drogas, han cambiado a través de los años. La clásica definición de vasorreactividad requería de la caída de la presión media de la arteria pulmonar (PAPM) y de la resistencia vascular pulmonar (RVP) superior al 20\% respecto del basal ${ }^{4}$. Dicha definición ha sido criticada en los últimos años debido a la falta de predicción en el tiempo de quienes realmente se benefician en forma sostenida con el uso de BCC. En el mayor estudio efectuado al respecto, en una serie francesa de 557 pacientes con HAP, usando los criterios clásicos de vasorreactividad, sólo el 12,6\% de los pacientes resultó positivo y en el seguimiento a largo plazo, el $50 \%$ de ellos sostuvo el beneficio ${ }^{10}$. Esto llevó a que en el consenso de la OMS del año $2003^{11}$ se redefiniera vasorreactividad por la caída de la PAPM superior a $10 \mathrm{mmHg}$ respecto al basal, alcanzando un nadir, en número absoluto, inferior a $40 \mathrm{mmHg}$, con un débito cardíaco estable. Si bien esta nueva definición no cuenta con estudios prospectivos que la sustenten, parece razonable en la perspectiva de la terapia que se usará, exigir un nivel de cambio suficiente para que los pacientes alcancen cifras cercanas a la normalidad luego del test agudo. Estos nuevos criterios si bien son más estrictos, probablemente permitan restringir el uso de terapias vasodilatadoras como los $\mathrm{BCC}$ a pacientes con HAP con clara y significativa respuesta aguda a vasodilatadores.

En Chile se desconoce la prevalencia de vasorreactividad en los pacientes con HAP y su respuesta a la terapia con $\mathrm{BCC}$.

En el Instituto Nacional del Tórax (INT) se ha implementado un programa de HAP para la evaluación y tratamiento de estos pacientes, en el contexto del cual se lleva a cabo esta investigación.

El objetivo del presente estudio fue evaluar de manera prospectiva a los pacientes con HAP que responden al test agudo con vasodilatadores y su respuesta clínica, funcional y hemodinámica a la terapia con BCC.

\section{Material y Método}

A partir del año 2003, todos los pacientes con sospecha clínica y ecocardiográfica de HAP son evaluados en el Instituto Nacional del Tórax por un equipo multidisciplinario compuesto por un médico broncopulmonar, dos cardiólogos, un reumatólogo y un kinesiólogo. Todos los pacientes son sometidos a una ecocardiografía efectuada por expertos y si la presión arterial sistólica pulmonar (PAPS) es superior a 40 $\mathrm{mmHg}$ y no existe otra causa que explique la hipertensión pulmonar, son programados para un estudio hemodinámico $(\mathrm{EH})$ mediante cateterismo derecho, para confirmar el diagnóstico y evaluar la respuesta aguda a adenosina, el vasodilatador usado para medir la vasorreactividad.

Fueron incluidos en el presente estudio los pacientes con HAP demostrada mediante $\mathrm{EH}$, que se encontraban sintomáticos y sin terapia específica para la HAP al momento de la evaluación diagnóstica. Los pacientes con hipertensión pulmonar de otro origen, diferente al grupo I de la $\mathrm{OMS}^{5}$ tales como los asociados a enfermedades pulmonares crónicas, a falla valvular o ventricular izquierda, a embolia pulmonar crónica entre otras, fueron excluidos del estudio.

Todos los pacientes incorporados en el estudio firmaron consentimiento informado y el protocolo fue aprobado por el Comité de Ética del Servicio de Salud Metropolitano Oriente.

\section{Evaluación basal}

Todos los pacientes son evaluados al momento del diagnóstico del punto de vista clínico, 
funcional y hemodinámico. La evaluación clínica es objetivada mediante la graduación de capacidad funcional (CF) de la OMS (I a IV) según presenten disnea, fatiga, dolor torácico o síncopes $^{6,7}$. Además la disnea es objetivada mediante la puntuación de la escala de disnea de Borg (0 a 10). El test de caminata de 6 minutos (DR6) que mide la distancia recorrida en metros en 6 minutos es efectuada siempre por el mismo operador, siguiendo las normas del consenso de la ATS $2002^{12}$.

\section{Mediciones hemodinámicas}

El EH mediante cateterismo derecho permite medir la PAPM, la RVP y la resistencia vascular indexada según superficie corporal (RVPI), el índice cardíaco (IC) y la saturación de arteria pulmonar (SATP).

El test de adenosina se efectúa mediante la administración endovenosa en infusión continua de adenosina en dosis crecientes cada 2 minutos a partir de $50 \mu \mathrm{g} / \mathrm{kg} / \mathrm{min}$ hasta $250 \mu \mathrm{g} / \mathrm{kg} /$ min. El test se detiene si existen efectos molestos para el paciente tales como cefalea, vómitos o compromiso hemodinámico sistémico (hipotensión o arritmias).

El criterio de vasorreactividad usado fue el clásico de reducción de $20 \%$ o más en la PAPM y RVP con débito cardíaco estable.

\section{Tratamiento}

Los pacientes respondedores al test de adenosina (vaso-reactivos) son tratados con diltiazem en dosis entre $60-120 \mathrm{mg}$ cada 8 horas asociado a terapia anticoagulante y diurética, esta última a juicio clínico del equipo. En el caso de deterioro clínico o funcional significativo (progresión negativa en uno o más grados en la clase funcional asociado a caída superior al $15 \%$ en la distancia recorrida en el DR6 o signos de falla ventricular derecha), el paciente es reevaluado por el equipo para incremento de dosis o eventual cambio de terapia.

\section{Seguimiento}

Los pacientes son evaluados a nivel basal mediante la puntuación de clase funcional $(\mathrm{CF})$, escala de disnea de Borg, DR6, EH y test de Adenosina. La evaluación clínica y funcional se repite en los meses 6-12 y luego anualmente. El $\mathrm{EH}$ es repetido al $6^{\circ}$ mes de terapia. El día del segundo EH los pacientes son advertidos de suspender la terapia anticoagulante 3 días antes del examen y concurrir en ayuno.

\section{Estadística}

Para el análisis estadístico se usó el programa STATA $^{\mathrm{tm}}$ versión 10 . Los datos numéricos continuos son expresados en promedios y desviaciones estándar. Para las variables ordinales como la escala de disnea de Borg, se usó el cálculo de medianas.

Las comparaciones entre variables continuas, de distribución normal según el test de Shapiro, fueron efectuadas mediante el test de student pareado de doble cola. Para variables repetidas como la distancia recorrida en diferentes momentos del seguimiento, se usó el análisis de medidas repetidas con metodología de estimación de GEE (generalized equation estimation) con matriz de covarianzas intercambiables. Un $\mathrm{p}<0,05$ fue considerado significativo.

\section{Resultados}

En el período 2003-2008, hubo 54 pacientes con HAP demostrada mediante EH. De ellos, 6 pacientes $(11 \%$; $95 \%$ IC, $4 \%$ al $22 \%)$ fueron vaso-reactivos en el test de adenosina. Ningún paciente presentó efectos adversos durante el procedimiento.

Las características basales de los 6 pacientes se ilustran en la Tabla 1. Todos los pacientes son mujeres jóvenes, la mayoría con HAP idiopática en clase funcional III, con una HAP moderada a severa según las variables hemodinámicas evaluadas en el momento del diagnóstico.

Tabla 1. Características basales de los 6 pacientes con hipertensión arterial pulmonar con test de vasorreactividad positivo

\begin{tabular}{ll}
\hline Características & \\
\hline Sexo & 6 mujeres \\
Edad & $45 \pm 14$ años \\
Etiología & 4 idiopáticas; 1 AR; 1E \\
Capacidad funcional (II:III:IV) $2: 4: 0$ \\
Borg & 4 \\
DR6 & $399 \pm 68 \mathrm{~m}$ \\
PAPM & $60,3 \pm 6 \mathrm{mmHg}$ \\
IRVP & $1.886 \pm 464$ \\
IC & $2,3 \pm 0,6 \mathrm{~L} / \mathrm{min} / \mathrm{m}^{2}$ \\
\hline
\end{tabular}

Cada valor representa el promedio \pm su desviación estándar. AR: Artritis reumatoide; E: Síndrome de Eisenmenger; DR6: Distancia recorrida en el test de caminata de 6 minutos; PAPM: Presión de arteria pulmonar media; IRVP: Índice de resistencia vascular pulmonar, en dinas $\bullet s^{\bullet} \mathrm{cm}^{-5}$; IC: índice cardíaco. 
Tabla 2. Variables hemodinámicas de los 6 pacientes vasorreactivos durante el estudio diagnóstico mediante el test de adenosina

\begin{tabular}{lcccc}
\hline Pacientes & PAPM basal & $\begin{array}{c}\text { PAPM post } \\
\text { adenosina }\end{array}$ & $\begin{array}{c}\text { \% reducción } \\
\text { PAPM }\end{array}$ & $\begin{array}{c}\text { \% reducción } \\
\text { IRVP }\end{array}$ \\
\hline 1 & 51 & 34 & 33 & 38 \\
2 & 70 & 44 & 37 & 33 \\
3 & 60 & 45 & 25 & 42 \\
4 & 62 & 46 & 26 & 35 \\
5 & 58 & 45 & 22 & 28 \\
6 & 61 & 36 & 45 & 60 \\
Promedio & 60,3 & 41,8 & 31 & 39 \\
\hline
\end{tabular}

PAPM: Presión de arteria pulmonar media en $\mathrm{mmHg}$; IRVP:Índice de resistencia vascular pulmonar en dinas ${ }^{\bullet} \mathrm{s}^{\bullet} \mathrm{cm}^{-5}$.

La magnitud de caída de la PAPM y RVP en el test de adenosina se ilustra en la Tabla 2. En ella se aprecia que en promedio los pacientes descienden 31 y $39 \%$ la PAPM y RVP respectivamente alcanzando cifras absolutas de PAPM post adenosina, en promedio de $41,8 \mathrm{mmHg}$.

En el curso de la terapia, todos los pacientes refieren una mejoría en su condición clínica funcional. Dos pacientes mejoran a CF I y ningún paciente se encuentra en CF IV al $3^{\circ}$ año de seguimiento. Respecto a la condición basal, en que 2:4:0 pacientes se encontraban en CF II:III y IV, al $3^{\circ}$ año, mejoran a 2:3:1 en CF I:II y III respectivamente.

La escala Borg mejoró de una mediana de 4 a nivel basal a 3 a los 6 meses, 1 a los 12 meses, 2 a los 24 meses y 0 a los 36 meses $(p=0,052)$.

La distancia recorrida (DR) aumentó en prome- dio al mes 6-12-24 en 78,8 $\pm 62,4 \mathrm{~m}(\mathrm{p}=0,03)$, $82,8 \pm 63,9 \mathrm{~m}(\mathrm{p}=0,02)$ y $87 \pm 57,9 \mathrm{~m}(\mathrm{p}=0,01)$ como se ilustra en la Figura 1. A los 36 meses, la ganancia de la DR es de $40 \pm 118 \mathrm{~m}(\mathrm{p}=$ $0,4)$. Una paciente al $3^{\circ}$ año deterioró su test de caminata de 6 minutos. En el tiempo se observó una ganancia promedio de 3 metros por mes de seguimiento hasta el $2^{\circ}$ año, situación que luego no se sostiene debido a que hay una paciente que deterioró su condición funcional.

Todas las variables hemodinámicas mejoraron al $6^{\circ}$ mes respecto al basal como se ilustra en las Figuras 2, 3 y 4. La PAPM disminuyó de $60,3 \pm 6$ a $45,3 \pm 13 \mathrm{mmHg}(\mathrm{p}=0,018)$, al igual que la IRVP, que se modificó de $1.886 \pm$ 464 a $1.097 \pm 554$ dinas $\bullet \mathrm{s}^{\bullet} \mathrm{cm}^{-5}(\mathrm{p}=0,018)$. El IC mejora de $2,32 \pm 0,6$ a $3,1 \pm 1,5 \mathrm{~L} / \mathrm{min} / \mathrm{m}^{2}$ $(\mathrm{p}=0,2)$.

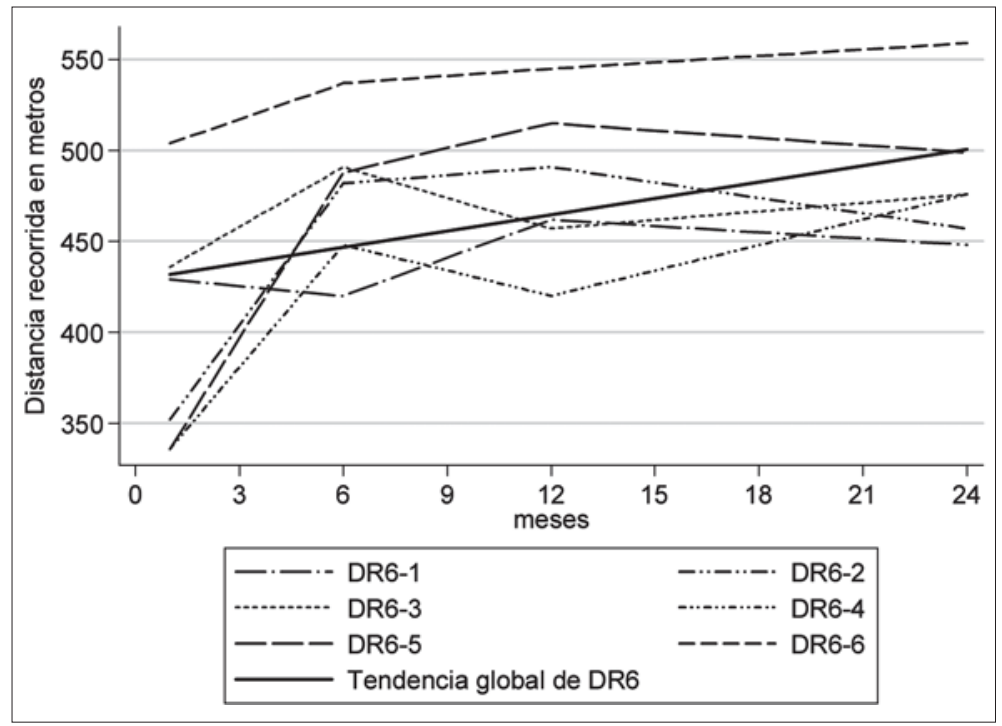

Figura 1. Evolución de la distancia recorrida en 6 minutos (DR6) en promedio y la de cada uno de los 6 pacientes en diferentes momentos del seguimiento. La línea central representa la tendencia promedio de todos los pacientes. Hasta los 2 años de seguimiento, la velocidad de ganancia es de $3 \mathrm{~m}$ por mes (coeficiente $=2,99$ ). Una paciente deterioró su condición funcional al $3^{\text {er }}$ año por lo que no fue posible efectuar este análisis en tal momento. Hasta los 24 meses, los pacientes incrementan significativamente la distancia recorrida $(\mathrm{p}=0,003)$. 

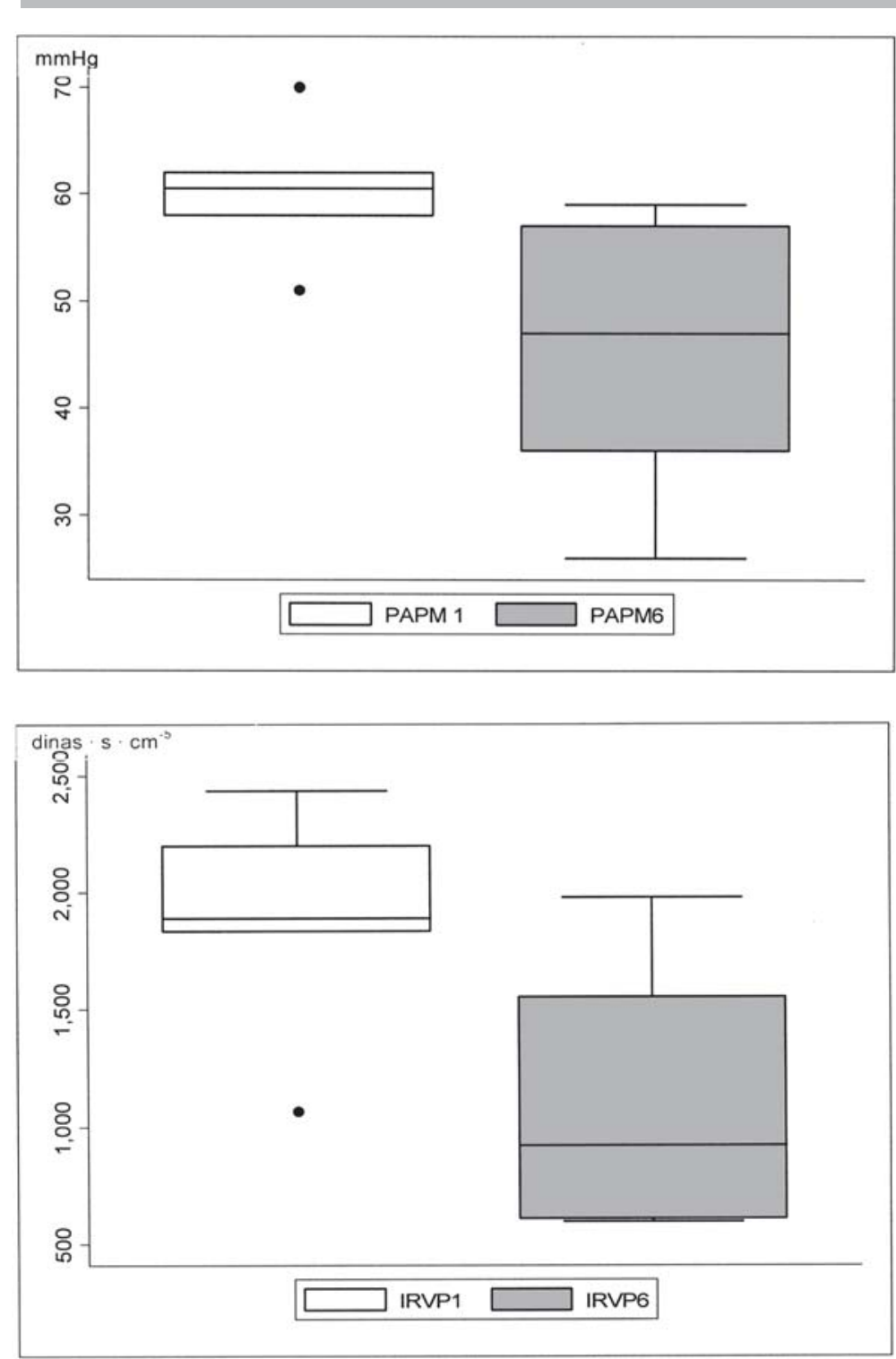

Figura 2. Evolución de la presión arterial pulmonar media (PAPM) desde el nivel basal (PAPM 1), en el momento del estudio hemodinámico diagnóstico y luego de 6 meses de terapia con diltiazem (PAPM 6). La caída de la PAPM es significativa $(\mathrm{p}=0,018)$. En el gráfico de box plot ("cajas"), la línea dentro de la caja indica la mediana de la variable y los bordes inferior y superior indican el percentil 25 y 75 respectivamente. Las líneas verticales externas a la caja indican la dispersión de la variable como recorrido intercuartílico y los puntos en negro, aislados, fuera de las cajas, indican valores fuera de los rangos esperados.

Figura 3. Evolución de la resistencia vascular pulmonar indexada (RVPI) desde el nivel basal (IRVP1), en el momento del estudio hemodinámico diagnóstico y luego de 6 meses de terapia con diltiazem (IRVP6). La caída de la resistencia es significativa $(\mathrm{p}=0,018)$. Los detalles del gráfico (box plot) son los mismos que los señalados en la figura 2.

Figura 4. Evolución del IC (índice cardíaco) desde su condición basal (IC1), en el momento del estudio hemodinámico diagnóstico y luego de 6 meses de terapia con diltiazem (IC6). Se observa un aumento del índice cardíaco, que no alcanzó significación estadística $(\mathrm{p}=0,2)$.

Los detalles del gráfico ("box plot") son los mismos que los señalados en la figura 2. 
Es importante destacar que la PAPM promedio alcanzada luego de 6 meses de terapia $(45,4$ $\mathrm{mmHg}$ ), es muy similar a la PAPM promedio alcanzada posterior a la infusión de adenosina $(41,8 \mathrm{mmHg})$.

Todos los pacientes se encuentran vivos en la evaluación del $3^{\text {er }}$ año si bien una paciente deterioró su condición funcional fundamentalmente en el DR6 por lo que requirió terapia específica adicional con sildenafil, encontrándose estable en las evaluaciones posteriores.

\section{Discusión}

La prevalencia de pacientes con HAP y test de vasorreactividad positiva fue del $11 \%$ en la serie estudiada y es similar a lo comunicado en la literatura ${ }^{10}$. Si bien el criterio de vasorreactividad utilizado fue el clásico de caída de la presión y resistencia pulmonar superior al $20 \%$, que era el criterio vigente a nivel mundial al momento de iniciar este estudio, los pacientes alcanzan luego de la adenosina, cifras muy cercanas a $40 \mathrm{mmHg}$, que forma parte del criterio actual de vasorreactividad junto con la caída mayor a $10 \mathrm{mmHg}$ en la PAPM, que todos los pacientes cumplen. Si bien los pacientes fueron considerados vaso-reactivos según el criterio clásico, se acercaron estrechamente a la definición actual ${ }^{11}$. Además, la cifra de PAPM alcanzada luego de 6 meses de terapia con diltiazem es similar a la obtenida luego del test de adenosina, lo que sugiere un buen nivel de predicción entre el test efectuado y la respuesta farmacológica objetivada en el tiempo.

Los pacientes vasorreactivos tienen un nivel de gravedad moderado a grave según sus variables funcionales y hemodinámicas. Cabe destacar que estos pacientes tienen características epidemiológicas similares a los pacientes no vasorreactivos reportados por nuestro grupo en una caracterización de los pacientes con $\mathrm{HAP}^{13}$ lo que aleja la posibilidad de enfrentar un grupo muy diferente en términos de edad, etiología o gravedad al momento del diagnóstico. Cabe destacar que en la serie se incluyó un paciente con Síndrome de Eisenmenger. Su inclusión se debe a que cumplió ampliamente con los criterios de vasorreactividad descritos y no se diferenció del resto del grupo en términos de evolución, respuesta a la terapia o efectos adversos. Por otro lado, en la evaluación hemodinámica del sexto mes, demostró una respuesta similar a la evidenciada en el estudio basal durante el test de respuesta aguda a vasodilatadores lo que fundamenta una buena respuesta a BCC.
En el período de seguimiento de 2 años, los pacientes vasorreactivos tratados con diltiazem se beneficiaron del punto de vista clínico, funcional y hemodinámico en forma significativa, demostrando que han sido seleccionados adecuadamente y que correspondían a verdaderos respondedores.

Respecto a la ganancia neta en la DR6, es importante destacar que esta es superior a la publicada con el uso de medicamentos específicos para la HAP, tales como sildenafil, el medicamento más ampliamente disponible en nuestro medio. El estudio Super- $1^{14}$ demostró un incremento al $6^{\circ}$ mes y al año de 48 a $50 \mathrm{~m}$. Cifras similares se han comunicado con prostaciclinas y bloqueadores de los receptores de endotelinas de modo que este aumento en la distancia recorrida con la terapia con BCC incluso supera a terapias específicas de primera línea utilizados en los pacientes no vasorreactivos ${ }^{15}$. Cabe destacar que todas estas terapias son de mayor costo, toxicidad y de menor disponibilidad que los BCC. Todo lo anterior enfatiza la importancia de efectuar un EH con test de vasorreactividad en la evaluación inicial de los pacientes con HAP, ya que la pesquisa del grupo de pacientes respondedores permite a este selecto grupo, ofrecer una terapia económica, segura y ampliamente disponible que le permitirá un beneficio clínico, funcional y hemodinámico, con una sobrevida superior a la del grupo no vaso-reactivo ${ }^{16,17}$. Debido al mejor pronóstico y evolución asociada a la condición de vaso-reactivo, es que se recomienda efectuar esta prueba aguda con vasodilatadores en todos los centros especializados y de referencia en el estudio inicial de los pacientes con $\mathrm{HAP}^{7,15,16}$. Por otro lado, no se recomienda el uso empírico de $\mathrm{BCC}$ en pacientes con HAP sin vasorreactividad demostrada ya que podrían deteriorar el intercambio gaseoso alvéolo-capilar ${ }^{5}$. Tampoco parece racional el inicio de terapias empíricas tales como sildenafil o prostaciclinas, sin antes cerciorarse de no estar frente a pacientes respondedores a vasodilatadores $^{5}$. Por último, debido a que se desconoce en qué pacientes el beneficio será sostenido, es que se recomienda en todos los pacientes que inician terapia con $\mathrm{BCC}$, un seguimiento estricto del punto de vista clínico y funcional y en el caso de deterioro, probablemente objetivar la condición del punto de vista hemodinámico antes de iniciar una terapia específica alternativa.

En suma, los pacientes con vasorreactividad demostrada, tienen una evolución satisfactoria con la terapia con diltiazem en la evaluación clínica, funcional y hemodinámica por lo que se 
recomienda, a todo paciente con sospecha de de HAP, una evaluación en un centro especializado, mediante EH y test de vasorreactividad. En los pacientes respondedores a esta prueba aguda, la terapia con BCC es segura y efectiva.

\section{Agradecimientos}

A Mariam Torres, químico-farmacéutico, jefe de Farmacia del Instituto Nacional del Tórax por su eficiente gestión en la obtención y suministro de adenosina para los estudios de vasorreactividad y por su apoyo constante al programa de HAP del Instituto.

\section{Bibliografía}

1.- RICH S, DANTZKER D R, AYRES S M, BERGOFSKY E H, BRUNDAGE B H, DETRE K M, et al. Primary pulmonary hypertension: a national prospective study. Ann Intern Med 1987; 107: 216-23.

2.- D’ALONZO G E, BARST R J, AYRES S M, BERGOFSKY E H, BRUNDAGE B H, DETRE K M, et al. Survival in patients with primary pulmonary hypertension: results from a national prospective registry. Ann Intern Med 1991; 115: 343-9.

3.- MIYAMOTO S, NAGAYA N, SATOH T, KYOTANI S, SAKAMAKI F, FUJITA M, et al. Clinical Correlates and prognostic significance of six-minute walk test in patients with primary pulmonary hypertension. Am J Respir Crit Care Med 2000; 161 (2 Pt 1): 487-92.

4.- RICH S, KAUFMANN E, LEVY P S. The effect of high doses of calcium channel blockers on survival in primary pulmonary hypertension. N Engl J Med 1992; 327: 76-81.

5.- SIMONNEAU G, GALIÉ N, RUBIN L J, LANGLEBEN D, SEEGER W, DOMENIGHETTI G, et al. Clinical classification of pulmonary hypertension. J Am Coll Cardiol 2004; 43: 5S-12S.

6.- BRITISH CARDIAC SOCIETY GUIDELINES AND MEDICAL PRACTICE COMMITTEE, AND APPROVED BY THE BRITISH THORACIC SOCIETY AND THE BRITISH SOCIETY OF RHEUMATOLOGY. Recommendations on the Management of Pulmonary Hypertension in Clinical
Practice. Heart 2001; 86 (Suppl 1): i1-i13.

7.- MCGOON M, GUTTERMAN D, STEEN V, BARST R, MCCRORY D C, FORTIN T A, et al. American College of Chest Physicians. Screening, early detection, and diagnosis of pulmonary arterial hypertension. Chest 2004; 126 (Suppl 1): 14S.

8.- SITBON O, BRENOT F, DENJEAN A, BERGERON A, PARENT F, AZARIAN R, et al. Inhaled nitric oxide as a screening vasodilator agent in primary pulmonary hypertension. Am J Respir Crit Care Med 1995; 151 (2Pt1): 384-9.

9.- MORGAN J M, MCCORMACK D G, GRIFFITHS M J D, MORGAN C J, BARNES P J, EVANS $\mathrm{T}$ W. Adenosine as a vasodilator in primary pulmonary hypertension. Circulation 1991; 84: 1145-9.

10.- SITBON O, HUMBERT M, JAIS X, IOOS V, HAMID A M, PROVENCHER S, et al. Long-term response to calcium channel blockers in idiopathic pulmonary arterial hypertension. Circulation 2005; 111: 3105-11.

11.- GALIE N, TORBICKI A, BARST R, DARTEVELLE P, HAWORTH S, HIGENBOTTAM T, et al. Guidelines on diagnosis and treatment of pulmonary arterial hypertension. The Task Force on Diagnosis and treatment of Pulmonary Arterial Hypertension of the European Society of Cardiology. Eur Heart J 2004; 25: 2243-78.

12.- ATS statement guidelines for the six-minute walk test. ATS Committee on Proficiency Standards for Clinical Pulmonary Function Laboratories. Am J Respir Crit Care Med 2002; 166: 111-7.

13.- ZAGOLÍN M, WAINSTEIN E, URIARTE P, PARRA C. Clinical, functional and hemodynamic features of patients with pulmonary arterial hypertension. Rev Méd Chile 2006; 134: 589-95.

14.- GALIE N, GHOFRANI H A, TORBICKI A, BARST R J, RUBIN L J, BADESCH D, et al. Sildenafil Use in Pulmonary Arterial Hypertension (SUPER) Study Group.Sildenafil citrate therapy for pulmonary arterial hypertension. N Engl J Med 2005; 353: 2148-57.

15.- BADESCH D B, ABMAN S H, SIMONNEAU G, RUBIN L J, MCLAUGHLIN V V. Medical therapy for pulmonary arterial hypertension: updated ACCP evidence-based clinical practice guidelines. Chest 2007; 131: 1917-28.

16.- HOEPER M M, RUBIN L J. Update in pulmonary hypertension 2005. Am J Respir Crit Care Med 2006; 173: 499-505.

17.- RICH S, KAUFMANN E, LEVY P S. The effect of high doses of calcium-channel blockers on survival in primary pulmonary hypertension. New Engl J Med 1992; 327: 76-81.

Correspondencia a:

Mónica Zagolín B.

Instituto Nacional del Tórax.

Av. José Manuel Infante 717, Santiago, Chile.

E-mail: mzagolin@torax.cl 